\title{
THE GALACTIC ELECTRON \\ DISTRIBUTION FROM A PULSAR SURVEY
}

\author{
A. G. LYNE \\ University of Manchester, Nuffield Radio Astronomy Laboratories, \\ Jodrell Bank, Cheshire, United Kingdom
}

\begin{abstract}
The recent high-sensitivity pulsar survey at Jodrell Bank has allowed a statistical study of more distant objects. The longitude distribution suggests that many of the pulsars observed have distances greater than $5 \mathrm{kpc}$, leading to an upper limit of about $0.03 \mathrm{~cm}^{-3}$ for the mean electron density. The electron density averaged over distances of a few hundred parsecs seems to be very constant. The width of the electron distribution in the $z$-direction appears to be greater than about $600 \mathrm{pc}$.
\end{abstract}

One year ago about a total of 65 pulsars had been discovered by eight observatories, all observing with different instruments, frequencies and sensitivities. The search at Molonglo was the only survey which had a high sensitivity, a thorough coverage and well-defined selection effects. This enabled Large (1971) to study the distribution and statistical properties of pulsars on a sample of 29 objects, scattered over a large area of sky.

From this work it was possible to predict that with a greater sensitivity many, more distant pulsars would be observable at low galactic latitudes and it would be possible to study pulsar distribution on a much larger galactic scale. A survey of the galactic plane was therefore carried out at Jodrell Bank at $408 \mathrm{MHz}$ using the Mk I telescope. (Davies et al., 1972, 1973). The experiment used a 4-MHz bandwidth and consisted of on-line period analysis on 11-min sections of data taken at points in the search area.

During the survey, 51 pulsars were detected. An indication of the improvement in sensitivity over previous searches was that only 11 of these had been discovered at other observatories. I shall describe only a few of the results of the survey, namely those which have a bearing upon the general distribution of electrons in the Galaxy.

Figure 1 shows the area covered by the survey and the positions of the pulsars detected, superimposed on a contour map of the background brightness distribution at $400 \mathrm{MHz}$ (Seeger et al., 1966). There are a number of small selection effects which are present in our data, but in general these are well defined and can be taken into account in our analyses. The most important of these are the area and thoroughness of the search over the area, and the system sensitivity which varies over the sky because of the galactic continuum radio background. The main result of the latter is that a number of weak pulsars at low longitudes and latitudes could have been missed.

Figure 2 shows the longitude distribution of those pulsars within $5^{\circ}$ of latitude of the plane. The top diagram is the distribution corrected only for the thoroughness of coverage and the ordinate is the deduced number of pulsars observable per square degree. The lower diagram indicates the estimate of the true distribution after allowing

F. J. Kerr and S. C. Simonson, III (eds.). Galactic Radio Astronomy, 87-95. All Rights Reserved. Copyright $\bar{C} 1974$ by the IAU. 


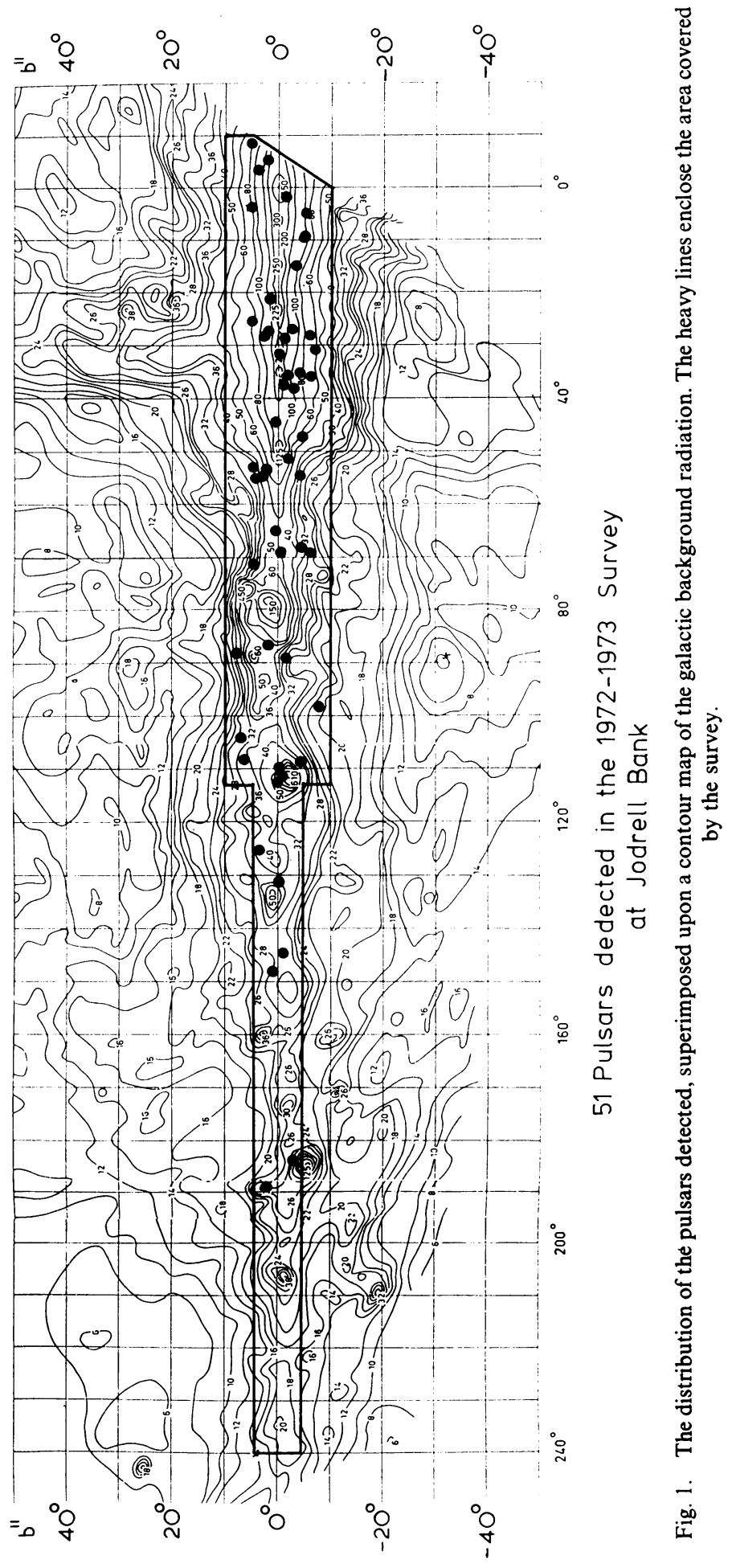


for system temperature variations over the sky under the assumption that the pulsars are a disc population. The justification for this will be seen shortly.

We see that there is a strong longitude concentration of pulsars, the bulk of them lying at longitudes of less than $100^{\circ}$. There is about a three to one ratio in densities
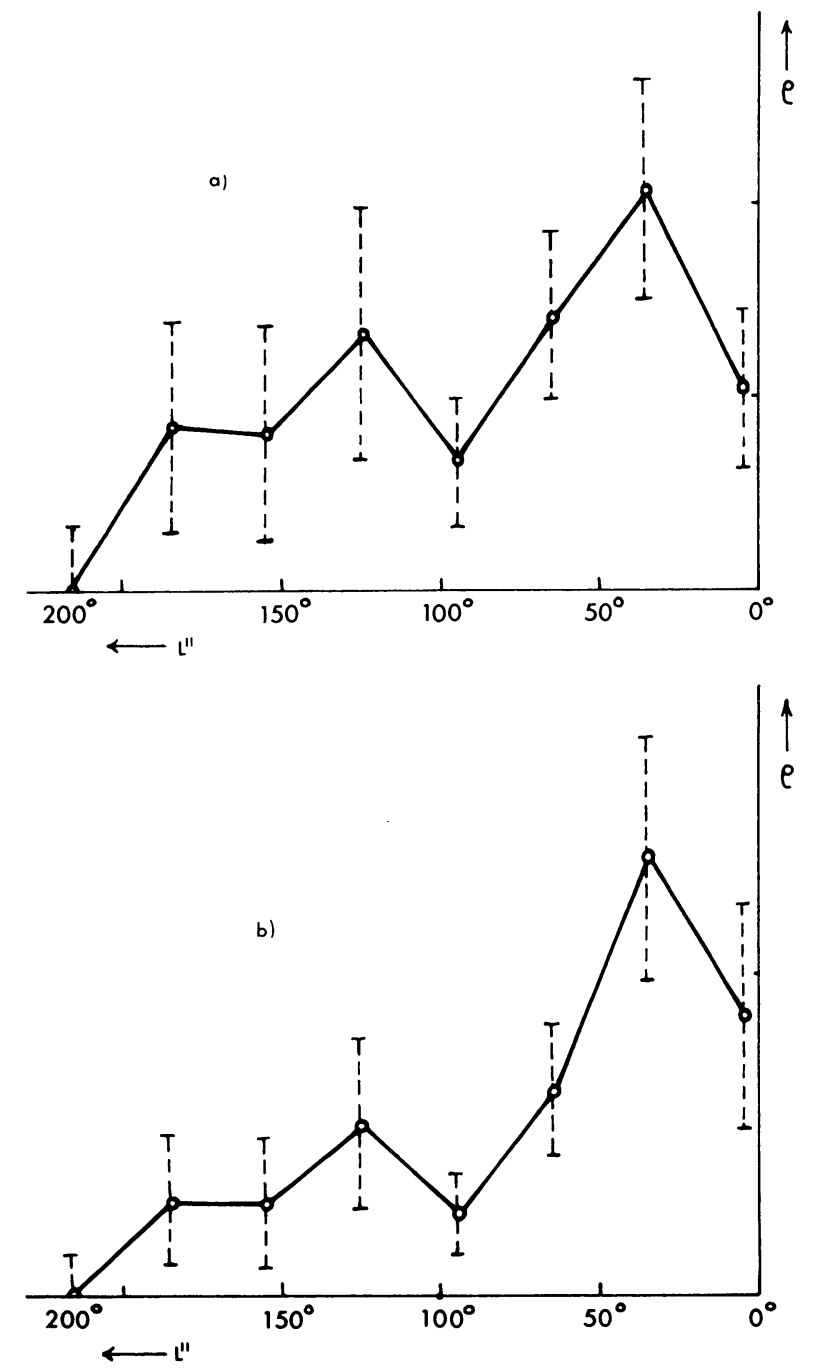

Fig. 2. (a) The observed longitude density of pulsars having $|b| \leqslant 5^{\circ}$ after correction for thoroughness of coverage. (b) The same as Figure $2 \mathrm{a}$ but after correction for variation of sky background temperature.

between the galactic centre and anticentre regions. This is clearly not just a local clustering due to the Sun's position with respect to the local spiral structure because of the observed narrowness of the latitude distribution indicated in Figure 3. Again, the top diagram is the observed density, while the lower one is that corrected for the background temperature. 


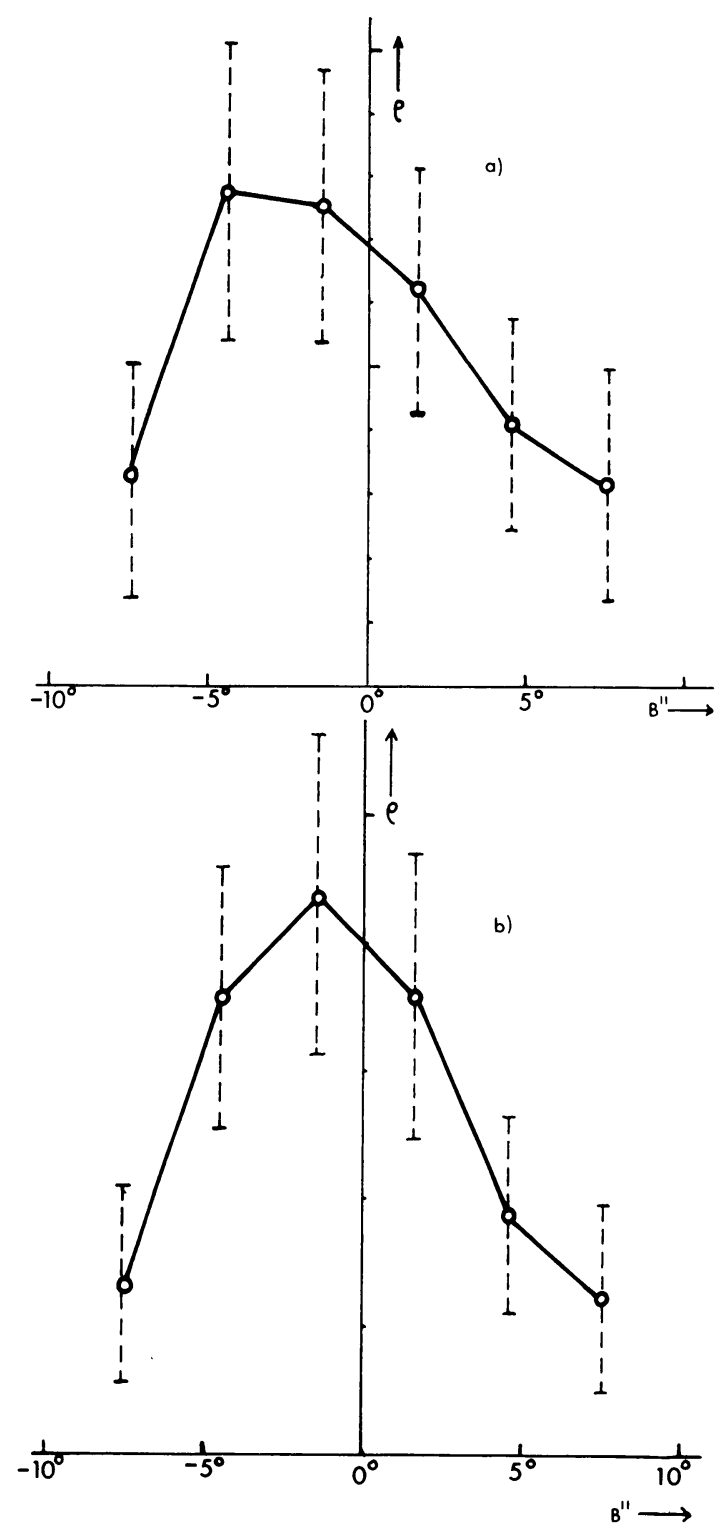

Fig. 3. (a) The observed latitude density of pulsars having $350<l<114^{\circ}$ after correction for thoroughness of coverage. (b) The same as Figure 3a but after correction for variation of sky background temperature.

We are therefore seeing pulsars on a galactic scale and to obtain the strong longitude dependence of their distribution, without putting any formal assumptions upon the way we might expect pulsars to be distributed through the Galaxy, I would estimate that on average they are at least half the distance of the galactic centre from the Sun, about $5 \mathrm{kpc}$ away. This conclusion is reached by considering the nature of the radial dependence of the densities of various galactic populations, in both our 
own Galaxy and in M31. (See for example Arp, 1964). This dependence is usually smooth and gradual. In the case of the OB associations observed in M31 (van den Bergh, 1964), there does seem to be quite a sharp fall-off in density near the outer regions of the Galaxy and if the Sun were situated near such a fall-off in the pulsar population, the pulsars might be generally a little closer than $5 \mathrm{kpc}$. However, there is no evidence for such an effect in any populations at the Sun's distance from the galactic centre. The mean dispersion measure of the pulsars is about $150 \mathrm{pc} \mathrm{cm}^{-3}$, leading to a rough estimate of $0.03 \mathrm{~cm}^{-3}$ for an upper limit for the mean electron density.

This is nicely compatible with the arguments presented by Guélin earlier. The value of the electron density might be refined further if we could identify the distribution with the spiral structure of the Galaxy. The clusters at longitudes $50^{\circ}$ and $30^{\circ}$ correspond quite closely to the tangential points of the Sagittarius and NormaScutum arms, suggesting that we are seeing some spiral structure. In fact, if a mean electron density of about 0.025 is assumed, there does seem to be quite a good correspondence between the pulsar population and the spiral structure, but with only about 50 pulsars, I do not consider the case to be very strong.

Having argued about the distribution of pulsars in the plane of the Galaxy, I would like to give a brief discussion of the distribution of pulsars above and below the plane in $z$ distance. As we will see, this leads to conclusions concerning the width of the ionised layer and its uniformity.

We can deduce a pulsar's $z$ distance from its dispersion measure under the assumption of a uniform extended ionized layer wider than the pulsar distribution. In Figure 4 we see how the $z$ distance so deduced and quoted in units of dispersion measure varies with distance away from the Sun. Of the 105 known pulsars, I have not included in this diagram 5 pulsars with poorly determined dispersion measures and the 14 pulsars found in low latitude surveys, namely the high-longitude Jodrell Bank pulsars (6) and the pulsars recently discovered at Parkes (8) (Komesaroff et al., 1973a, b), because these have a strong selection effect upon this diagram.

The Parkes pulsars, for instance, would mostly lie within the two lines marked $b= \pm 2^{\circ}$ and hence on this diagram would all be necessarily low $z$ distance objects. The latitude limitation of the main Jodrell Bank survey $\left(b<10^{\circ}\right)$ is not very serious, although it may give rise to a small excess of low $z$ distance local pulsars. In fact many of these pulsars were discovered in other, wider-latitude surveys suggesting that many of the higher $z$ distance local pulsars missed by the Jodrell Bank survey would have been discovered and are included in the diagram.

I find the most remarkable thing about this diagram is the constancy of the mean distance of pulsars off the plane as a function of distance away from the Sun at least out to dispersion measures of about 200. Table I shows how the mean distance off the plane, $\langle|z|\rangle$, varies with the distance, $D$, along the plane, both quoted in units of dispersion measure. The errors are the standard errors.

This presentation of the data simply demonstrates quantitively how well the pulsar dispersion measures obey the $\operatorname{cosec} b$ law. 


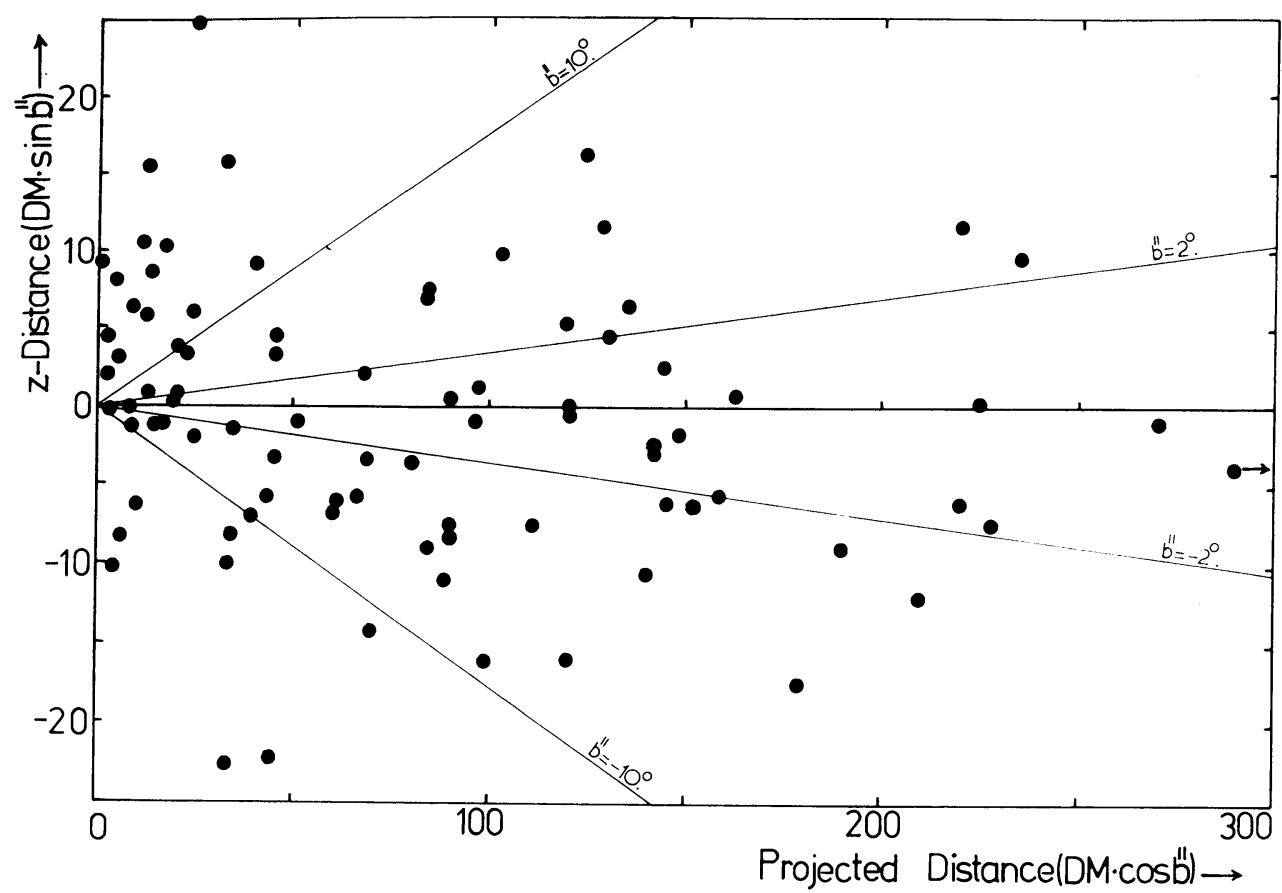

Fig. 4. The derived $z$-distances of pulsars plotted against the distances along the galactic plane from the Sun. The units of both quantities are in units of dispersion measure, $\mathrm{pc} \mathrm{cm}^{-3}$.

TABLE I

Mean distances of pulsars from the plane

\begin{tabular}{rl}
\hline$D\left(\mathrm{pc} \mathrm{cm}^{-3}\right)$ & $\langle|Z|\rangle\left(\mathrm{pc} \mathrm{cm}^{-3}\right)$ \\
\hline $0-50$ & $6.8 \pm 0.8$ \\
$50-100$ & $6.2 \pm 0.8$ \\
$100-150$ & $6.5 \pm 1.0$ \\
$>150$ & $7.0 \pm 1.1$ \\
\hline
\end{tabular}

If the line of sight to any pulsar intercepted a large $\mathrm{H}$ il region, then the apparent $z$ distance could be increased considerably. The data is thus consistent with a very simple model of the distribution of electrons and pulsars, in which the electrons have a uniform density out to a distance of about $6 \mathrm{kpc}$ from the Sun, and large $\mathrm{H}$ in regions contribute only a minor amount to the dispersion measures of pulsars in a statistical sense. In particular, the mean electron density over distances of this order is very similar to that immediately in the Sun's neighbourhood. Prentice and ter Haar (1969) and others from a more local selection of pulsars have come to similar conclusions for distances out to about 1 or $2 \mathrm{kpc}$. 
Finally, Figure 5 shows the pulsar $z$ distribution. This shows a substantially monotonic fall in numbers with $z$ distance. If the ionised layer is substantially thinner than the pulsar layer, this would show a peak at a non-zero $z$ distance. The absence of this effect suggests therefore that the ionised layer has a full width of greater than about $600 \mathrm{pc}$ in the Sun's neighbourhood.
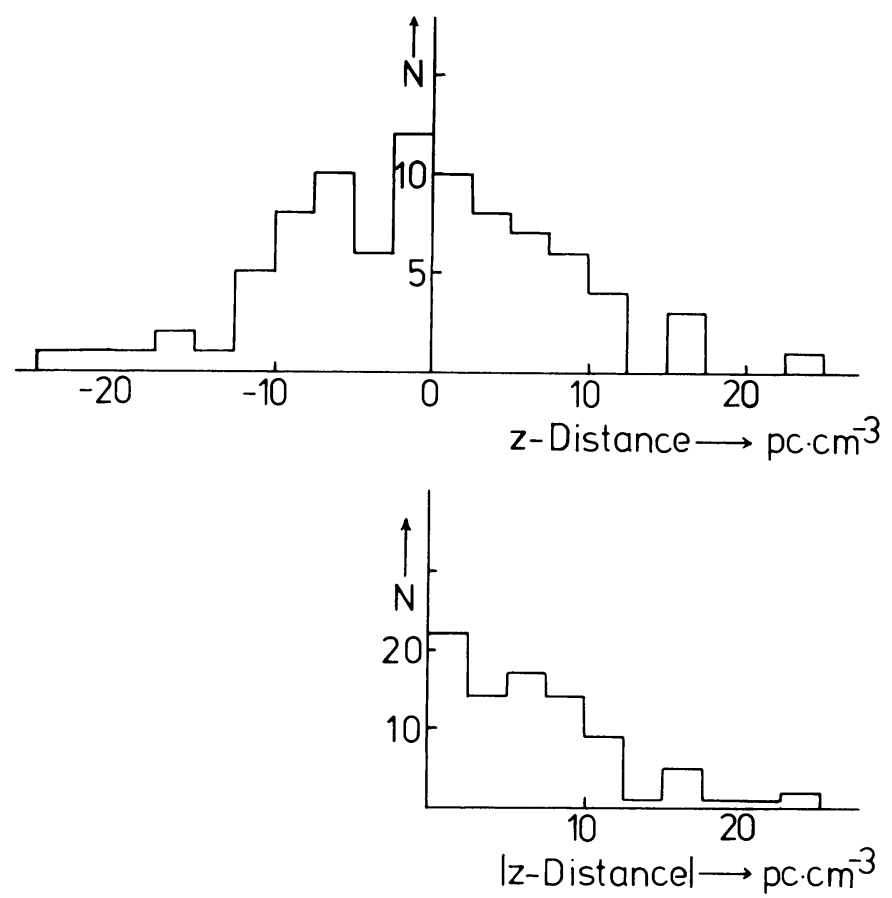

Fig. 5. Histograms of the numbers of pulsars as a function of the $z$-distance and modulus of the $z$-distance in units of $\mathrm{pc} \mathrm{cm}^{-3}$.

The deduced width of the pulsar distribution depends upon the precise form of the electron distribution. Whatever this is, the observed width of the $z$ distribution in Figure 5 and an assumed mean electron density of $0.03 \mathrm{~cm}^{-3}$ lead to a full-width to half-power points of at least $460 \mathrm{pc}$ for the pulsar distribution.

\section{References}

Arp, H.: 1964, Astrophys. J. 139, 1045.

Bergh, S. van den: 1964, Astrophys. J. Suppl. 9, 65.

Davies, J. G., Lyne, A. G., and Seiradakis, J. H. : 1972, Nature 240, 229.

Davies, J. G., Lyne, A. G., and Seiradakis, J. H.: 1973, Nature 244, 84.

Komesaroff, M. M., Hamilton, P. A., McCulloch, P. M., Ables, J. G., and Cooke, D. J.: 1973a, IAU Circ. No. 2505.

Komesaroff, M. M., Hamilton, P. A., McCulloch, P. M., Ables, J. G., and Cooke, D. J. : 1973b, IAU Circ. No. 2563.

Large, M. I. : 1971, in R. D. Davies and F. G. Smith (ed.), 'The Crab Nebula', IAU Symp. 46, 165. 
Prentice, A. J. R. and ter Haar, D. : 1969, Monthly Notices Roy. Astron. Soc. 146, 423.

Seeger, Ch. L., Westerhout, G., Conway, R. G., and Hoekama, T.: 1965, Bull. Astron. Inst. Neth. $18,11$.

\section{A. G. Lyne}

University of Manchester,

Nuffield Radio Astronomy Laboratory,

Jodrell Bank, Cheshire SK11 9DL, United Kingdom

\section{DISCUSSION}

Guélin: You have said that you can see correlations, taking $n_{\mathrm{e}}=0.025 \mathrm{~cm}^{-3}$ for the pulsars, with the spiral structure. I have made that calculation, and I don't find it. I think I have taken all the pulsars. I mean that the distribution is just chance along these directions.

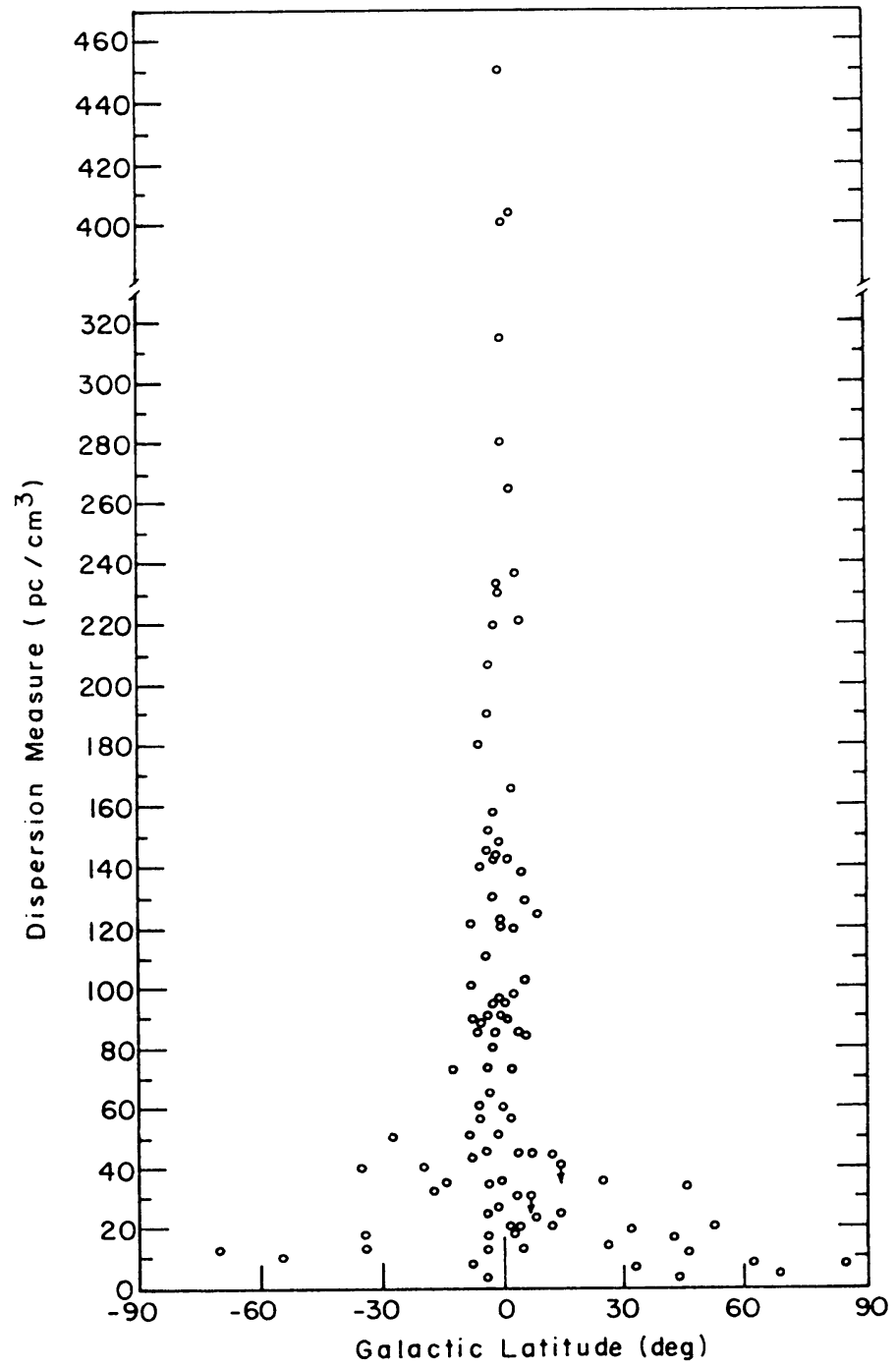

Fig. 1. Pulsar dispersion measures vs galactic latitude as of August 1973 (Terzian). 
Lyne: Yes, I do not consider the case to be very strong. There is an indication of it; I don't believe it is more than that.

Van Woerden: As a matter of definition: one may use $D M \sin b$ and $D M \cos b$ for estimates of vertical and horizontal distances, but I think it inadvisable to call these quantities 'distances.'

Terzian: In Figure 1 I would like to show the up-to-date data (August 1973) on pulsar dispersion measures shown as a function of galactic latitude; 105 pulsars are included.

F. G. Smith: Many of the 105 pulsars have been found in surveys with strong selection effects. To establish a sample of 51 pulsars it was necessary to extend the survey into relatively non-productive areas of the sky, in contrast to surveys which looked only in the best places. 